\title{
Non-steady Global Magnetic Fields in Kinematic Theory
}

\author{
J.H.G.M. van Geffen ${ }^{1,2}$, P. Hoyng ${ }^{1}$, C. Zwaan² \\ ${ }^{1}$ Laboratory for Space Research, Sorbonnelaan 2, 3584 CA Utrecht \\ ${ }^{2}$ Astronomical Institute, P.O. Box 80000,3508 TA Utrecht
}

\begin{abstract}
The dynamo equation for the mean field $\langle B\rangle$ contains a random forcing term of unknown magnitude, which is therefore always omitted. The influence of this term is potentially large. To evaluate its effect, we employ ensemble averaging. If an ensemble average is used, there is no random forcing term in the dynamo equation. The effect of fluctuations is that the ensemble members get out of phase, so that $\langle\boldsymbol{B}\rangle \rightarrow 0$. The damping time of $\langle\boldsymbol{B}\rangle$ can be found by requiring that the mean energy $\langle\boldsymbol{B} B\rangle$ remains finite. The eigenvalues $\kappa$ of the dynamo equation then all have negative real parts. Im $\kappa$ determines the period, and $-\operatorname{Re} \kappa / \operatorname{Im} \kappa$ the relative period stability of the dynamo. We have developed a code to solve the equation for $\langle\boldsymbol{B B}\rangle$ in a spherical shell (the convection zone), assuming axisymmetry. We report our first results, which do not yet include differential rotation. Using spherically symmetric boundary conditions, we reproduce the well known $\alpha^{2}$-dynamo, whose behaviour is known analytically. For instance, for an $\alpha^{2}$ dynamo located in a shell with inner boundary at $R / 2$, we find that $\langle\boldsymbol{B} B\rangle$ remains finite for $R^{2} \gamma / \beta=1.48$, where $\beta$ represents turbulent diffusion and $\gamma$ turbulent vorticity. Taking $\alpha=1 / 4(\beta \gamma)^{1 / 2}$ - a factor of four below maximum helicity - implies that we have a dynamo number $C_{\alpha} \equiv R \alpha / \beta=0.30$. Using this value we find a damping time of $6 \times 10^{-2} R^{2} / \beta$ for $\langle B\rangle$, which is a measure for the coherence time of $B$ in a single ensemble member. This result implies that the large-scale field of this particular $\alpha^{2}$-dynamo reorganizes its structure completely on a time scale of only about one year (for solar values of $R$ and $\beta$ ), and it shows the enormous influence of random forcing in general.
\end{abstract}

\section{Introduction}

The effects of turbulence in the convection zone of stars on the magnetic field $B$ are described by the dynamo equation:

$$
\partial_{t}\langle\boldsymbol{B}\rangle=\alpha \nabla \times\langle\boldsymbol{B}\rangle+\beta \nabla^{2}\langle\boldsymbol{B}\rangle,
$$

where $\langle\boldsymbol{B}\rangle$ is some average of the magnetic field $\boldsymbol{B}$, which we discuss presently. We have assumed that there is no differential rotation. The first term in (1) is the turbulent helicity term, and the second is the turbulent diffusion term. Their coefficients are defined as:

$$
\alpha \approx-\left\langle\boldsymbol{u}_{1} \cdot \nabla \times \boldsymbol{u}_{1}\right\rangle \tau_{c} / 3 ; \quad \beta \approx\left\langle u_{1}^{2}\right\rangle \tau_{c} / 3,
$$

which we assume to be constant; $u_{1}(r, t)$ is the turbulent velocity, and $\tau_{c}$ its correlation time.

All finite solutions of the dynamo equation are strictly periodic, and in our case this period is infinite, since we assume no differential rotation. Strict periodicity is very strange, since the underlying mechanisms are of a stochastic nature, so we 
would expect to find at best quasi-periodic solutions. Yet, the traditional dynamo equation suggests that the dynamo has an infinite memory. The origin of this feature lies in the derivation of (1). The averaging operator $\langle\cdot\rangle$ must satisfy the Reynolds relations (e.g., Krause and Rädler, 1980):

$$
\begin{aligned}
& \langle f+g\rangle=\langle f\rangle+\langle g\rangle ; \quad\langle f\langle g\rangle\rangle=\langle f\rangle\langle g\rangle ; \quad\langle c\rangle=c ; \\
& \langle\cdot\rangle \text { commutes with } \nabla, \partial_{t} \text { and all integrals }
\end{aligned}
$$

where $f(\boldsymbol{r}, t)$ and $g(\boldsymbol{r}, t)$ are arbitrary functions and $c$ is an arbitrary constant.

The average over space or time, generally used in (1), does not satisfy (3b) and (3d), which means that there is actually an extra term on the r.h.s. of (1) to account for this discrepancy. This extra term depends on the turbulent velocity $\boldsymbol{u}_{1}$ and has therefore the nature of a random forcing term, henceforth abbreviated as r.f.t. Even if $\langle\cdot\rangle$ is defined as a longitudinal average, i.e. $\langle\bullet\rangle=(2 \pi)^{-1} \oint\langle\bullet\rangle d \phi$, which satisfies (3) exactly, there still is random forcing, because according to their definition $\alpha$ and $\beta$ will now have a fluctuation component according to their definition (2).

The r.f.t. is usually omitted, and that explains the (unphysical) infinite phase memory of the dynamo. We wish to investigate the magnitude of this r.f.t., but since it is very difficult to obtain a manageable expression for it, we adopt another approach.

\section{Ensemble average}

We interpret $\langle\cdot\rangle$ in (1) as an ensemble average, which does obey (3) exactly. In this case $\alpha$ and $\beta$ are constant, meaning that (1) holds as it stands: there is no r.f.t. if an ensemble average is used. One has to take the ensemble literally: an infinite set of identical systems, each having a different realization of $u_{1}(r, t)$. This, however, implies that $\langle\boldsymbol{B}\rangle$ no longer refers to a specific dynamo, or in other words: we don't know which of the ensemble members is our dynamo. Nevertheless, the ensemble averaging turns out to be a useful approach for estimating the order of magnitude of the r.f.t.

Our line of thought is as follows: Since each of the ensemble members has a different realization of fluctuations, the ensemble members gradually get out of phase. In other words: eventually $\langle\boldsymbol{B}\rangle \rightarrow 0$. If we can determine the damping time of this process, we know the correlation time of $\boldsymbol{B}$ itself in a given ensemble member.

\section{Mean magnetic energy}

In order to find the damping time of $\langle B\rangle$, we analyse the mean magnetic energy $\langle\boldsymbol{B} \boldsymbol{B}\rangle$. On physical grounds we must require that this mean magnetic energy remains finite. For if $\langle\boldsymbol{B} \boldsymbol{B}\rangle \rightarrow 0$, then $B=0$ in the end, contrary to the assumption that there is a dynamo. And if $\langle\boldsymbol{B} B\rangle$ would increase, the energy would grow indefinitely, which is physically not acceptable either. Therefore we must require that $\langle\boldsymbol{B} \boldsymbol{B}\rangle$ remains finite. We can now find the damping time of $\langle\boldsymbol{B}\rangle$, because the requirement " $(\boldsymbol{B} \boldsymbol{B}\rangle$ remains finite" fixes a combination of parameters also present in the dynamo equation.

The equation for the tensor $T_{i j}=\left\langle B_{i} B_{j}\right\rangle$ is (Knobloch, 1978; Hoyng, 1987): 


$$
\partial_{t} T_{i j}=\alpha\left(\epsilon_{i k l} \nabla_{k} T_{l j}+\epsilon_{j k l} \nabla_{k} T_{l i}\right)+\beta \nabla^{2} T_{i j}+\frac{2}{5} \gamma\left(2 T_{k k} \delta_{i j}-T_{i j}\right),
$$

where $i, j$ represent Cartesian coordinates; recall that differential rotation is omitted. The first and second terms in (4) are turbulent helicity and diffusion, also present in the dynamo equation (1). The third term depends on the turbulent vorticity, and it represents generation of magnetic energy. The coefficient is defined as:

$$
\gamma \approx\left\langle\left|\nabla \times u_{1}\right|^{2}\right\rangle \tau_{c} / 3
$$

which we also assume to be constant. Note that $\alpha, \beta$ and $\gamma$ must fulfill Schwarz' inequality:

where:

$$
\alpha^{2} \leq \beta \gamma \quad \text { or } \quad C_{\alpha}^{2} \leq C_{\gamma}
$$

$$
C_{\alpha} \equiv R \alpha / \beta ; \quad C_{\gamma} \equiv R^{2} \gamma / \beta,
$$

with $R$ the outer radius of the dynamo. $C_{\alpha}$ is the usual dynamo number, important in both (1) and (4); $C_{\gamma}$ is a second "dynamo number" relevant for (4) only. The definition of these two numbers follows upon the introduction of a dimensionless time $t \beta / R^{2}$ in both (1) and (4).

The damping time of $\langle B\rangle$ is found as follows. We choose a value of $C_{\alpha}$ and determine, subject to condition (6), the value of $C_{\gamma}$ for which eq. (4) has a solution that remains finite. With the adopted value of $C_{\alpha}$ we determine the eigenvalues of (1), from which we obtain the damping time of $\langle B\rangle$. This is expounded in the following sections.

\section{Results}

We have developed a code to determine the eigenvalues of (4) in a spherical shell, using spherical coordinates $r, \theta, \phi$, and assuming axisymmetry. Eq. (4) is in fact a set of six coupled differential equations, and therefore six boundary conditions are needed, both on the inner boundary $r=r_{c}$ and on the outer boundary $r=R$; for completeness they are summarized in the Appendix.

Let us write the eigenvalues of (4) as $\Lambda_{k}=\lambda_{k}+\mu_{k} \mathrm{i}(k=0,1,2, \ldots)$. The real parts $\lambda_{k}$ of the eigenvalues form a decreasing series (because (4) is a parabolic equation). The requirement that $\langle B B\rangle$ remains finite implies that the largest of the real parts must equal zero, i.e. $\lambda_{0}=0$. The eigenfunction $T_{i j, 0}$ then describes the corresponding energy distribution within the dynamo; $T_{i j, 0}$ depends on the value of $C_{\alpha}$, but $\lambda_{0}$ does not (Van Geffen and Hoyng, 1990). Figure 1 shows two examples:

a) Spherically symmetric boundary conditions, which reproduces the spherically symmetric $\alpha^{2}$-dynamo, whose behaviour is known analytically (Van Geffen and Hoyng, 1990), and can serve as a check on the computations.

b) Axisymmetric boundary conditions. In this case there is a preferred direction (the vertical axis).

In both figures, the top row shows the distribution of magnetic energy along the radial direction $\left\langle B_{r}^{2}\right\rangle$, the bottom row along the $\phi$ direction $\left\langle B_{\phi}^{2}\right\rangle$. Since there is no magnetic field within the core, we have $B_{r}=0$ at the inner boundary, implying 
that $\left\langle B_{r}^{2}\right\rangle$ equals zero at $r=r_{c}$, and increases outward. On the other hand, $\left\langle B_{\phi}^{2}\right\rangle$ is maximal on the inner boundary and decreases outward.

$\left\langle B_{r}^{2}\right\rangle$

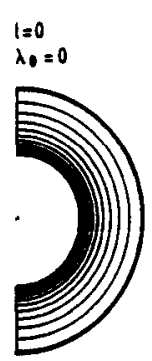

$\left\langle B_{4}^{2}\right\rangle$

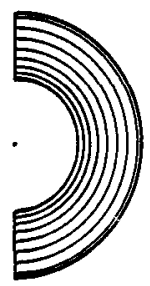

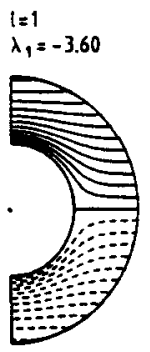

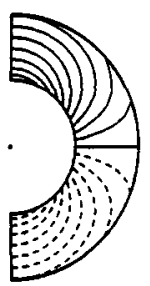

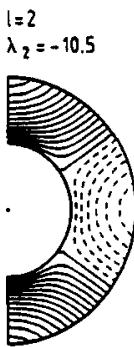

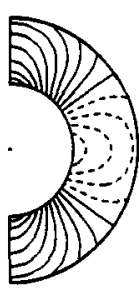

$\left\langle B_{r}^{2}\right\rangle$

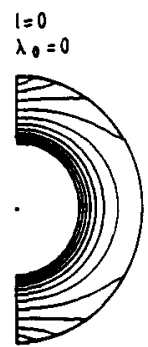

$\left\langle B_{\varphi}^{2}\right\rangle$

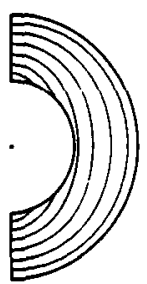

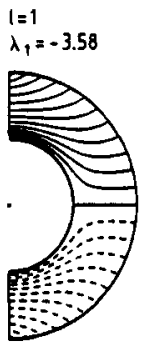

$1=2$

$\lambda_{2}=-10.5$
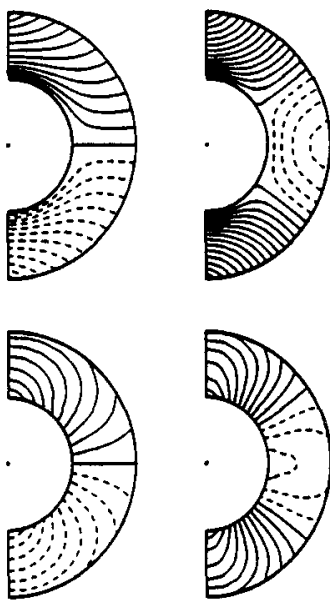

Fig. 1. Contour lines of the distribution of magnetic energy in a spherical shell, using a) spherically symmetric and b) axisymmetric boundary conditions. Solid lines represent positive contours, broken lines negative contours. In both cases $C_{\gamma}=R^{2} \gamma / \beta=$ 1.48, $C_{\alpha}=1 / 4 \sqrt{C_{\gamma}}=0.30$. See the text for an explanation.

The left column shows the eigenfunction $T_{i j, 0}$ belonging to the eigenvalue with the largest real part $\lambda_{0}=0$. The second and third column show the eigenfunctions belonging to the states with the next two largest real parts: $\lambda_{1}$ and $\lambda_{2}$. The solutions shown in Figure 1 are non-periodic, i.e. they have $\mu_{k}=0$. The states with negative energy $(k=1,2, \ldots)$ are damped and will play only a transient role, as the solution of (4) relaxes from arbitrary initial conditions to the stationary solution. Some of the higher eigenfunctions are periodic (that is: they have $\mu_{k} \neq 0$ ) in addition to being damped; we found, however, that $\mu_{0}$ always equals zero.

We can assign quantum numbers to the eigenmodes. First of all $m$ equals zero because of axisymmetry. The quantum number $l$, representing the number of zeros along the $\theta$ direction, takes values 0,1 and 2 as indicated in Figure 1. The radial quantum number $n$ equals zero in the cases shown, but going to larger values of $\left|\lambda_{k}\right|$ there will also be states with $n=1,2, \ldots$.

\section{Damping time of $\langle B\rangle$}

It turns out that $\lambda_{0}=0$ is reached if $C_{\gamma}=1.48$, independent of $C_{\alpha}$ (see Van Geffen and Hoyng, 1990). In the figure we have taken $C_{\alpha}=1 / 4 \sqrt{C_{\gamma}}=0.30$, so that $\alpha$ equals $1 / 4$ of its maximum value according (6). With this value we solve (1) analytically by extending the method of Krause and Rädler (1980, Ch. 14), to allow for the existence of an inner boundary (where $B_{r}=0$ ). The eigenvalues $\kappa_{k}$ of (1) are real, since there is no differential rotation. For $C_{\alpha}=0.30$ it turns out 
that $\kappa_{k}<0$ for all $k$, thus confirming that all solutions of the dynamo equation are damped (cf. Hoyng, 1987). The longest living mode is determined by the the smallest $\left|\kappa_{k}\right|$, which we found to be equal to $16 \beta / R^{2}$. Hence the damping time of $\langle B\rangle$ is $\left(16 \beta / R^{2}\right)^{-1} \approx 6 \times 10^{-2} R^{2} / \beta$.

Our interpretation of this result is as follows. The dynamo equation (1) with $\langle\cdot\rangle$ is a spatial or time average contains a r.f.t. and may be written as follows:

$$
\partial_{t}\langle\boldsymbol{B}\rangle=\alpha \nabla \times\langle\boldsymbol{B}\rangle+\beta \nabla^{2}\langle\boldsymbol{B}\rangle+\underbrace{\cdots\langle\boldsymbol{B}\rangle}_{r . f . t} .
$$

The diffusion term is of the order of $\beta / R^{2}$, so we must conclude that in this case the r.f.t. is about 16 times larger than the diffusion term. Although this is only a rough estimate, it shows that the r.f.t. is certainly not small, as has been assumed so far (e.g., Krause and Rädler, 1980). We have analyzed values of $C_{\alpha}$ ranging from 1 to $1 / 128$ times the maximum value $\sqrt{C_{\gamma}}$, and we found the damping time to be of the same order of magnitude as in the above example.

\section{Concluding remarks}

We have shown above that by using ensemble averaging and requiring that $\langle\boldsymbol{B} B\rangle$ remains finite, the damping time of $\langle B\rangle$ can be found, and the magnitude of the random forcing term in the dynamo equation can be estimated. We have also shown that this random forcing dominates in (8), rather than being negligible. This result raises several issues:

- First of all the role of non-linearities. Our main result is that random forcing is an essential ingredient in the dynamo equation (1), if an average over space or time is used. The use of an ensemble average to estimate the magnitude of the r.f.t. is merely a technical matter. Apparently the r.f.t. is very large and this makes the dynamo unsteady and erratic. At present it is not clear whether non-linearities (which are of course important) will further destabilize the dynamo. The traditional approach, however, where non-linear effects are included in (1) - for example by making $\alpha$ a function of $|\langle B\rangle|$ - while random forcing is ignored, seems rather pointless in the light of the present results.

- We have assumed isotropic, homogeneous turbulence and infinite resistivity. These assumptions are not very realistic and have to be removed, but we believe that this will not drastically change the outcome of our study.

- What happens if we adopt a larger value of $C_{\alpha}$ ? We found the dipole solution of (1) to be marginally stable only at $C_{\alpha}=6.57$. We have verified that for this large value of $C_{\alpha}$, eq. (4) always has growing solutions (i.e. $\lambda_{0}>0$ ). A full discussion will appear later.

- The aim of our work is to study the effect of random forcing in the solar dynamo, in order to find out whether the observed period stability of the 22-year cycle of the sun $(\delta P / P \approx 0.1)$ can be explained within linear theory.

Acknowledgements: We thank Dr. K.-H. Rädler and Dr. A. Brandenburg for many stimulating discussions. This research has been supported by the Netherlands Foundations for 
Astronomical Research (ASTRON) and the Space Research Organization of the Netherlands (SRON). Both organizations are supported by the Netherlands Organizations for Scientific Research (NWO). The computations are financed by the Foundation SURF from the National Fund for the use of Supercomputers (NFS).

\section{Appendix: Boundary conditions}

We consider a dynamo in a spherical shell: $0<r_{c} \leq r \leq R$, and assume axisymmetry. The first column shows the boundary conditions and quantities specified on the inner boundary, and the second column those on the outer boundary. The first two lines follow directly from physics, where $\epsilon \equiv 2 \mathrm{gem}$ is the mean magnetic energy density, and where $\sigma, \tau$ are related to the distribution of magnetic energy over spatial scales; these are discussed in more detail in Van Geffen and Hoyng (1990). The bottom three lines represent cross-correlation functions at the boundaries. On the axis: $T_{r \theta}=T_{r \phi}=T_{\theta \phi}=0, T_{\theta \theta}=T_{\phi \phi}$ because of axisymmetry.

\begin{tabular}{ll}
\hline$r=r_{c}$ & $r=R$ \\
\hline$\partial \epsilon / \partial r=0$ & $\partial \epsilon / \partial r+\epsilon / \varrho=0$ \\
$T_{r r}=T_{r \theta}=T_{r \phi}=0$ & \\
$\tau^{0}=T_{\theta \theta} / T_{\phi \phi}$ & $\sigma=\left(T_{\theta \theta}+T_{\phi \phi}\right) / T_{r r}$ \\
& $\tau=T_{\theta \theta} / T_{\phi \phi}$ \\
& $C_{r \theta}=T_{r \theta} / \sqrt{T_{r r} T_{\theta \theta}}$ \\
$C_{\theta \phi}^{0}=T_{\theta \phi} / \sqrt{T_{\theta \theta} T_{\phi \phi}}$ & $C_{r \phi}=T_{r \phi} / \sqrt{T_{r r} T_{\phi \phi}}$ \\
\hline
\end{tabular}

The distribution and correlation functions chosen for the graphs in Figure 1 are:

\begin{tabular}{ccc}
\cline { 2 - 3 } & spherically sym. & axisymmetric \\
\hline$\sigma$ & $1 / 2$ & $1 / 2+\sin \theta$ \\
$\tau^{0}, \tau$ & 1 & $1+\sin \theta$ \\
$C_{\theta \phi}^{0}, C_{\theta \phi}$ & 0 & $\sin \theta$ \\
$C_{r \theta}, C_{r \phi}$ & 0 & 0 \\
$R / \varrho$ & 1 & 1 \\
\hline
\end{tabular}

\section{References}

Hoyng, P.: 1987, Astron. Astrophys. 171, 357

Knobloch, E.: 1978, Astron. J. 220, 330

Krause, F., Rädler, K.-H.: 1980, Mean Field Magnetohydrodynamics and Dynamo Theory, Pergamon Press, London

Moffatt, H.K.: 1978, Magnetic Field Generation in Electrically Conducting Fluids, Cambridge University Press, Cambridge

Van Geffen, J.H.G.M., Hoyng, P.: 1990, Geophys. Astrophys. Fluid Dynam. 53, 109 